Brit. F. vener. Dis. (1973) 49, 301

\title{
Infection of the eye, genitalia, and rectum by a new serotype (Type G) of TRIC agent
}

\section{Clinical and laboratory aspects}

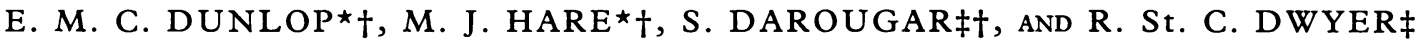 \\ From the Whitechapel Clinic ${ }^{\star}$, The London Hospital, The Diagnostic Clinict, Department of Clinical \\ Ophthalmology, Institute of Ophthalmology, and the Virus Laboratory $\ddagger$, Department of Clinical Ophthalmology, \\ Institute of Ophthalmology, University of London
}

The micro-immunofluorescence (micro-IF) test of Wang (Wang, 1971; Wang and Grayston, 1970; 1971; Treharne, Katzenelson, Davey, and Gray, 1971) provides a method for typing isolates of Chlamydia and for detecting the serological response by the patient to infection by the different serotypes. Six main serotypes (A to F) of TRIC agent have been described. The findings in the cases of five patients infected by a further serotype (Type $G$ ) identified by Treharne, Darougar, and Jones (1973) are now reported, together with those in a sixth epidemiologically related case in which the isolate was not typed but may well have been Type G.

\section{Patients and methods}

Chlamydia (Table), later shown to be TRIC agent Type G (Treharne and others, 1973), was isolated from the conjunctiva of a newborn baby (Baby JD) suffering from ophthalmia neonatorum due to TRIC agent, from the urethra of her father (Mr. JD) who was suffering from so called non-specific urethritis (NSU), from the cervix and the anorectal canal of her mother (Mrs. JD), from the urethra of a man (Mr. JO) who presented because of NSU, and from cervical material from one of his sexual partners (Mrs. JO(i)). Chlamydia was also isolated from cervical material from another of this man's sexual partners (Mrs. JO(ii)) but was not available for typing. The clinical methods of examination have been reported (Dunlop and others, 1964, 1965, 1969).

Address for reprints: E. M. C. Dunlop, as above

\section{Clinical findings}

BABY JD (Dunlop and others, 1969) developed 'sticky eyes' when 10 days old; she was treated with topical drops and ointment of unknown nature with little effect. When she was seen at the age of 9 weeks, her eyelids were swollen and there were hyperaemia, papillae, and follicles affecting the conjunctiva of the upper and lower lids of both eyes. There was congestion of the vulva and an excess of pus in vulval smears but this subsided spontaneously within 3 weeks. Proctoscopy and the examination of a rectal smear showed no abnormality.

TRIC agent Type $G$ was isolated from the conjunctiva but not from the vulva or rectum. Chlamydial inclusion bodies were detected in conjunctival smears by means of a direct fluorescent-antibody staining technique. $E$. coli was grown from urethra, vulva, and rectum, but no bacterial growth was obtained in cultures of material from the eyes. After treatment for 3 weeks with chlortetracycline eye ointment, the conjunctivae appeared normal. Intradermal tests with chlamydial antigens, namely Frei, acid-extract of lymphogranuloma venereum (LGV) agent, and an acid-extract of TRIC agent (Barwell, Dunlop, and Race, 1967) gave negative results.

The diagnosis was opthalmia neonatorum due to TRIC agent Type G. Review at the age of 3 years and 10 months showed no abnormality of the eyes or of the genital tract.

TABLE Source of six TRIC agent Type $G$ isolates and of an untyped chlamydial isolate

\begin{tabular}{|c|c|c|c|}
\hline \multicolumn{2}{|l|}{ Patient case code } & \multirow{2}{*}{$\frac{\text { Relationship }}{\text { Baby }}$} & \multirow{2}{*}{$\begin{array}{l}\text { Isolate } \\
\text { TRIC/G/GB/IOL-202/ON }\end{array}$} \\
\hline Ophthalmia neonatorum group & Baby JD & & \\
\hline & Mrs. JD & Mother & $\begin{array}{l}\text { TRIC } / G / G B / \text { IOL-201/G Cx } \\
\text { |TRIC } / G / G B / I O L-238 / R\end{array}$ \\
\hline 'NSU' group & $\begin{array}{l}\text { Mr. JO } \\
\text { Mrs. JO (i) } \\
\text { Mrs. JO (ii) }\end{array}$ & $\begin{array}{l}\text { Consort of Mrs. JO (i) and } \\
\text { Mrs. JO (ii) } \\
\text { Consort of Mr. JO } \\
\text { Consort of Mr. JO }\end{array}$ & $\begin{array}{l}\text { TRIC/G/GB/IOL-215/GU } \\
\text { TRIC/G/GB/IOL-241/G Cx } \\
\text { TRIC/ /GB/IOL-240 G Cx }\end{array}$ \\
\hline
\end{tabular}


MRS. JD (Dunlop and others, 1969), a Caribbean, was 22 years old when seen at the time that her baby was 9 weeks old. She had had vaginal discharge during her pregnancy; after delivery this was accompanied by lower abdominal pain made worse by marital intercourse when this was resumed. Examination with an operating microscope showed a mucopurulent cervical discharge, 'follicles' in the marginal area of the cervix, and some star-shaped areas of fibrosis (splash fibrosis). There were some pale slightly raised areas on the rectal mucosa; there was marked tenderness in the region of the left Fallopian tube on bimanual examination. The eyes appeared normal.

There was no enlargement of lymph nodes. There was a maximal count of 20 polymorphonuclear leucocytes per high-power $\left(\frac{1}{12}\right)$ field (HPF) in a Gram-stained urethral smear, and 30 in the cervical smear; no leucocytes were seen in the rectal smear. Isolates of TRIC agent Type G were obtained in cell culture from cervical scrapings and from a cervical swab, and a serologically identical agent was isolated from rectal material (Darougar, Kinnison, and Jones, 1971; Dunlop and others, 1971). Scanty growths of Staph. saprophyticus were obtained from urethra, cervix, vagina, and rectum, and $E$. coli was grown from the rectum. Cultures for $T$. vaginalis and Candida, serological tests for syphilis, four complement-fixation tests for group antichlamydial antibody (LGVCFT), an Ayre's smear, and intradermal tests for Chlamydia all gave negative results. Sera, taken before treatment and on three subsequent occasions, the last being 6 months after treatment, were tested by means of the micro-IF test. This showed reactivity against all serotypes, highest (titre 1 in 64) to TRIC agent D, E, G, and against LGV agent I, II, and III. The diagnosis was chlamydial infection of the genital tract and rectum, with salpingitis, due to TRIC agent Type G.

The patient was treated with oxytetracycline by mouth $250 \mathrm{mg}$. four times a day for 21 days. After this, signs of active inflammation had subsided and further tests for Chlamydia gave negative results.

MR. JD (Dunlop and others, 1969), a Caribbean bus-conductor, was 23 years of age when he was seen at the time that his daughter was 9 weeks old. For 5 months he had occasionally noticed urethral discharge in the mornings before passing urine. Over a year before attendance he had received three intramuscular injections of what he thought was penicillin from a family doctor as treatment for urethral discharge. There was some thin white urethral discharge after he had refrained from passing urine for 3 hours. A smear of this discharge contained over 100 polymorphonuclear leucocytes per HPF but no organisms. Culture gave a scanty growth of Staph. saprophyticus. Serological tests for syphilis and the LGVCFT gave negative results initially and on three further occasions during observation for 20 months. TRIC agent Type $G$ was isolated from urethral scrapings in cell culture. Intradermal tests for Chlamydia gave positive results: the Frei test gave $7 \mathrm{~mm}$. of induration, an acid-extract of LGV gave a negative result ( $5 \mathrm{~mm}$. of induration), but an acid-extract of TRIC agent gave $10 \mathrm{~mm}$. of induration. Sera taken before treatment and on three subsequent occasions were tested by means of the micro-IF test. This showed reactivity against all serotypes, highest (titre 1 in 32) to TRIC agent D, E, G, and to LGV III. He was treated with oxytetracycline by mouth $250 \mathrm{mg}$. four times a day for 21 days, followed by demethylchlortetracycline $300 \mathrm{mg}$. twice a day for 7 days, and then 'Tetrex' $500 \mathrm{mg}$. four times a day for 7 days because of low-grade persisting urethritis. After this he was symptom-free; urethral tests, examination of the prostato-vesicular fluid, and urethroscopy showed no abnormality.

$\mathrm{He}$ returned because of gonococcal urethritis 4 years after his first attendance. Although he again denied extramarital exposure, tests in the case of his wife did not show gonococci.

MR. JO (Dunlop and others, 1969), an unmarried Englishman, an upholsterer by trade, was 25 years old at the time of attendance. He had been treated 4 years earlier for NSU with one intramuscular injection of $1 \mathrm{~g}$. streptomycin and sulphamethoxypyridazine tablets by mouth for 5 days. He presented again because of a urethral discharge that he had had for 3 days. Tests showed that this was due to NSU. A smear of the urethral discharge contained a maximal count of 150 polymorphonuclear leucocytes per HPF after the patient had refrained from passing urine for 9 hours. TRIC agent Type $G$ was isolated in cell culture from urethral scrapings. A moderate growth of Staph. saprophyticus was obtained from material from the urethral meatus but no trichomonad or yeast was grown. Serological tests for syphilis gave negative results as did the LGVCFT initially and on three subsequent occasions. Sera taken before treatment and on two subsequent occasions were tested by means of the micro-IF test; this showed weak reactivity against all serotypes except TRIC agent B; the highest (titre 1 in 8 ) was against TRIC agent $A$. The patient was treated with oxytetracycline $250 \mathrm{mg}$. by mouth four times a day for 21 days with good response. The patient was seen five times after the completion of treatment, the last occasion being after 6 months had elapsed. On most 
occasions he had refrained from micturition for 4 hours before attendance; there was no urethral discharge, the urine was clear, the prostato-vesicular fluid was normal, and urethroscopy showed no abnormality. Two of this patient's sexual partners were examined.

MRS. JO (i), an Englishwoman separated from her husband, was 35 years old when examined. She had no abnormal symptoms. She had been treated 4 years earlier with streptomycin and a sulphonamide (as had Mr. JO) for non-specific genital infection after Mr. JO had developed NSU.

On examination there was an erosion of the marginal area of the cervix with areas of thickening and 'follicles' (Fig. 1); there was scarring near to the edge of the erosion. There was some mucus on an apparently normal anorectal mucosa. There was a maximal count of 5 polymorphonuclear leucocytes per HPF in the urethral smear, and 30 in the cervical smear; no leucocyte was seen in the rectal smear. Vaginal smears contained an excess of pus cells. TRIC agent Type $G$ was isolated from cervical scrapings in cell culture. Moderate growths of
$E$. coli and diphtheroids were obtained from the urethra and rectum, and scanty growths of Staph. saprophyticus and diphtheroids from the cervix and vagina, but no trichomonad or Candida. Serological tests for syphilis initially and on three subsequent occasions gave negative results.

Initially, and on four subsequent occasions, the LGVCFT was negative; on the fifth occasion, 6 months after treatment, it showed only partial fixation at a dilution of 1 in 4 . The micro-IF test showed reactivity against all serotypes, the highest (titre 1 in 32) being against TRIC agent $F$ and $G$ and against LGV agent II and III. A biopsy specimen initially showed endocervical tissue diffusely infiltrated with plasma cells and lymphocytes and containing a lymphoid follicle. An Ayre's smear gave a negative result.

Intradermal tests for Chlamydia were performed. The Frei test gave $7 \mathrm{~mm}$. of induration, an acidextract of LGV gave $12 \mathrm{~mm}$., an acid-extract of TRIC agent gave $9 \mathrm{~mm}$. Examination of the eyes showed a small scar in the conjunctiva of the left lower lid but no other abnormality.

The patient was treated with oxytetracycline

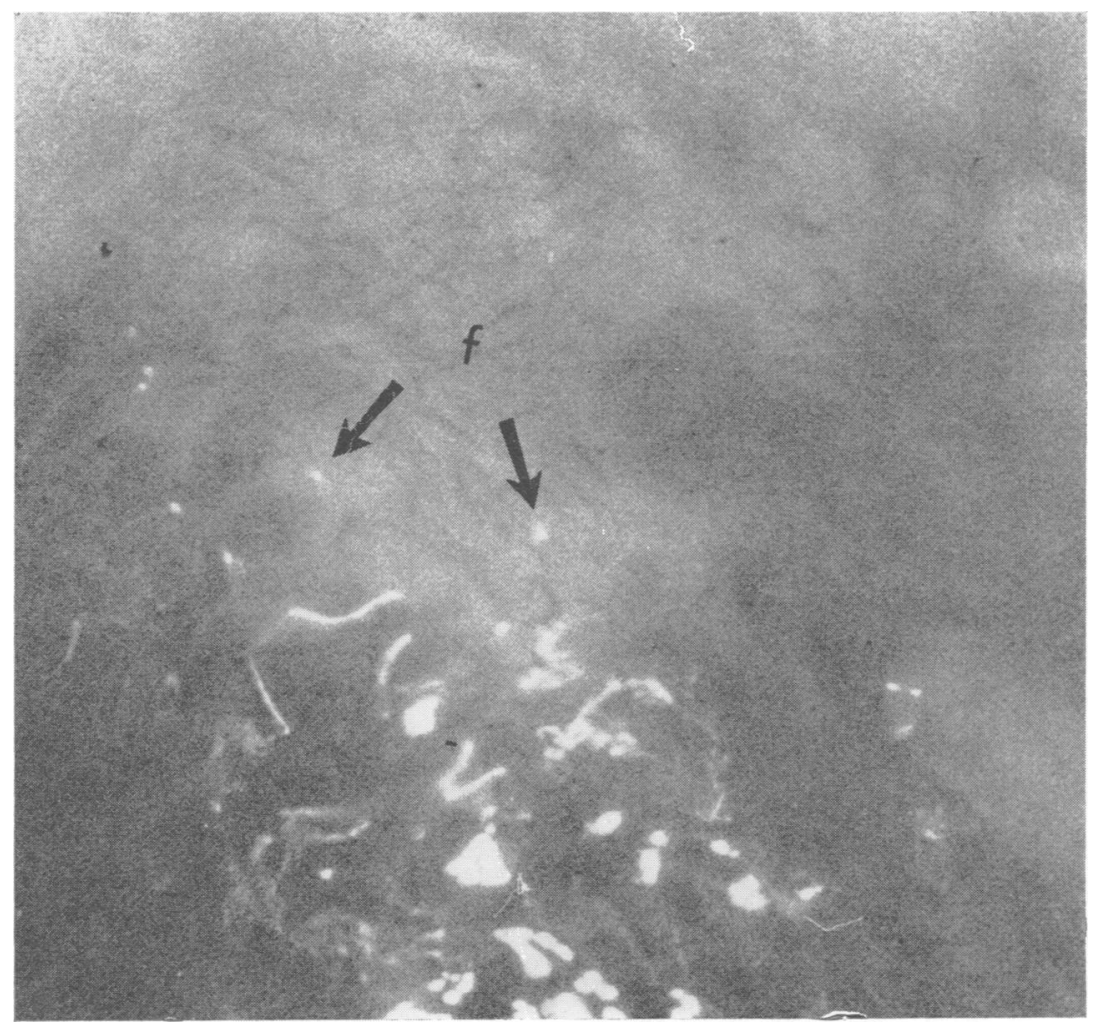

FIG. 1 Marginal area of cervix of Mrs. Fo (i), showing 'follicles' $(f), \times 6$ approx. 
$250 \mathrm{mg}$. four times a day by mouth for 21 days. After this tests for Chlamydia gave negative results. Apart from the development of vaginal candidiasis, which responded to nystatin pessaries, the clinical evidence of genital infection cleared.

MRS. JO (ii), a married English shop assistant, was 23 years old when she attended. She had had vaginal discharge for some months. She had had three attacks of 'cystitis', the last 2 years before attendance. She had first had sexual intercourse with $\mathrm{Mr}$. JO at the age of 17. Examination showed a congested erosion of the marginal area of the cervix which contained 'follicles' (Fig. 2). Examination of the eyes showed no abnormality. A maximal count of only six polymorphonuclear leucocytes per HPF was obtained in the cervical smear; only occasional leucocytes were present in the urethral smear, and none in the rectal smear. Serological tests for syphilis and an Ayre's smear gave negative results. Chlamydia was isolated in cell culture from cervical material but the isolate was not available for subsequent typing. No other specific organism was isolated. Intradermal tests for Chlamydia, serological tests for syphilis, and an Ayre's smear gave negative results. The LGVCFT showed complete fixation to 1 in 4 with partial fixation to 1 in 8 . The micro-IF test showed weak reactivity (titre 1 in 8 ) against all serotypes of TRIC agent and of LGV agent. It seems likely on epidemiological grounds that the Chlamydia isolated in this case was also TRIC agent Type G.

\section{Discussion}

Six of these seven isolates obtained in the six cases reported here have been identified as TRIC agent Type G. Each of the seven has been isolated from the genitalia or rectum or from eye disease associated with genital infection of the sort commonly caused by TRIC agent Types D, E, or F. The biological characteristics of this new Subgroup A chlamydial serotype have been reported by Treharne and others (1973).

The isolate from the rectum of Mrs. JD, in whose case there was no clinical sign of LGV, was used to inoculate the urethra of a baboon (Darougar and others, 1971) whence it was re-isolated after 14 days. At necropsy, inflammatory changes were present in

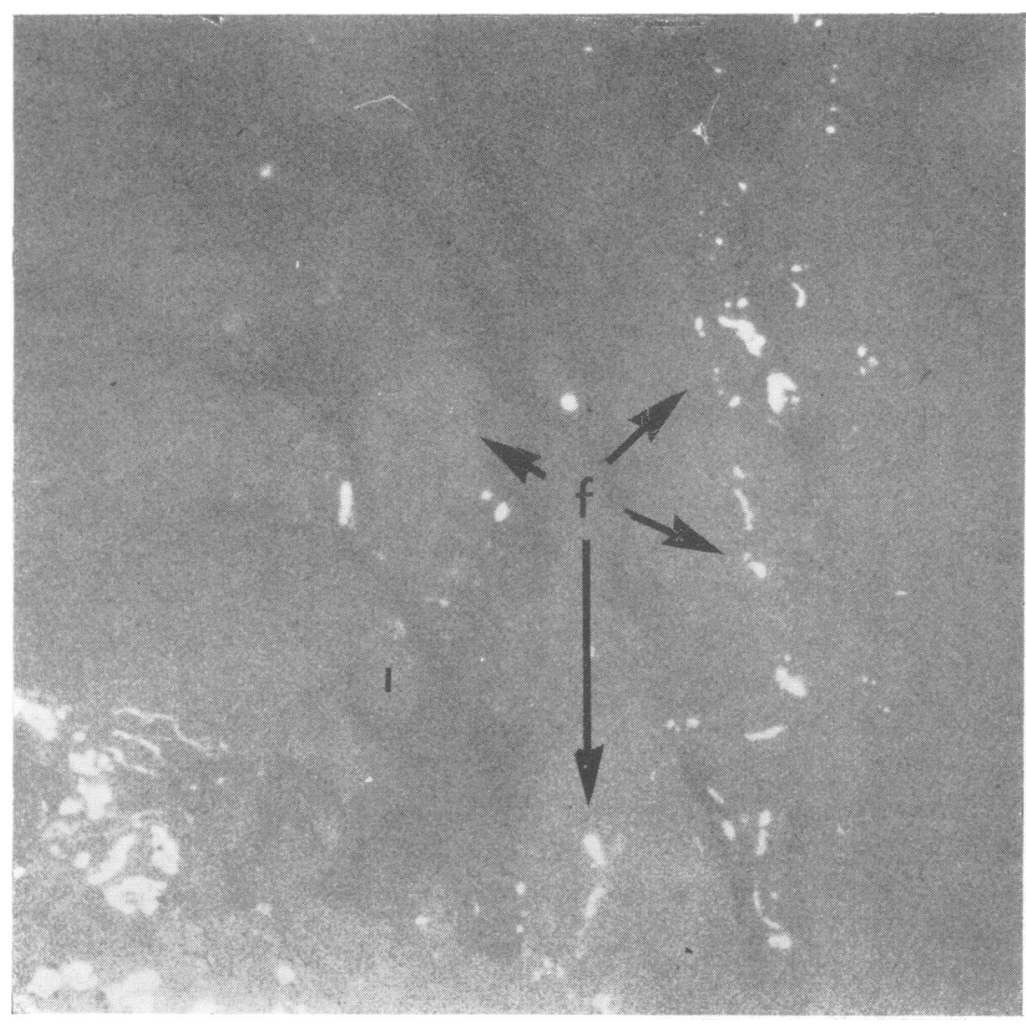

FIG. 2 Within marginal area of cervix of Mrs. $\mathcal{F O}$ (ii), showing 'follicles' $(f), \times 6$ approx. 
the urethra of the baboon. This agent also produced mild follicular conjunctivitis typical of TRIC agent after inoculation of the eyes of baboons (Darougar and others, 1971).

The seventh isolate, from the cervix of Mrs. JO (ii) may also have been TRIC agent Type G because that serotype had already been isolated from the urethra of a sexual partner ( $\mathrm{Mr}$. JO) and from the cervix of another consort of his (Mrs. JO (i)).

\section{Summary}

The findings are reported in the cases of five patients in two epidemiological groups (one related to ophthalmia neonatorum, the other to NSU) from whom six isolates were obtained of a newly-defined Subgroup A Chlamydia serotype: TRIC Type G.

In each case the agent was isolated from the genitalia or rectum or from an infected eye associated with genital infection, as occurs with TRIC agent Types D, E, or F.

We are grateful to our colleagues who referred patients to us for examination; to Dr. A. E. Wilkinson, Director of the VD Reference Laboratory, The London Hospital, for carrying out serological tests for syphilis, and cultures for bacteria, Trichomonas vaginalis, and Candida; to Prof. Norman Ashton, the Institute of Ophthalmology, for the histological studies; to the late Prof. C. F. Barwell, The London Hospital, for supplying antigens for intradermal tests and for carrying out the LGVCFT.

We thank The London Hospital and Moorfields Eye Hospital for financial support for clinical aspects of this study and the Departmental of Health and Social Security for financial support for the laboratory work on Chlamydia.

\section{References}

Barwell, C. F., Dunlop, E. M. C., and Race, J. W. (1967) Amer. F. Ophthal., 63, 1527
Darougar, S,. Kinnison, J. R., and Jones, B. R. (1971 In 'Trachoma and Related Disorders', ed. R. L. Nichols, p. 501. Excerpta Medica, Amsterdam

Dunlop, E. M. C., Al-Hussaini, M. K., Garland, J. A., Treharne, J. D., Harper, I. A., and Jones, B. R. (1965) Lancet, 1, 1125, 1286

-, Hare, M. J., Darougar, S., and Jones, B. R. (1971) In 'Trachoma and Related Disorders', ed. R. L. Nichols, p. 507. Excerpta Medica, Amsterdam

,,$---\longrightarrow$ and RICE, N. S. C. (1969) f. infect. Dis., 120, 463

, Jones, B. R., and Al-Hussaini, M. K. (1964) Brit. F. vener. Dis., 40, 33

Treharne, J. D., Darougar, S., and Jones, B. R. (1973) Ibid., 49, 295

—, Katzenelson, E., Davey, S. J., and Gray, S. J. (1971) In 'Trachoma and Related Disorders', ed. R. L. Nichols, p. 289. Excerpta Medica, Amsterdam

WANG, S.-P. (1971) Idem, p. 273

- and Grayston, J. T. (1970) Amer. F. Ophthal., 70, 367

(1971) In 'Trachoma and Related Disorders', ed. R. L. Nichols, p. 305. Excerpta Medica, Amsterdam.

Infection de l'oeil, des organes génitaux et du rectum par un nouveau sérotype (Type G) d'agent TRIC. Aspects cliniques et biologiques

\section{SOMMAIRE}

Chez 5 malades, se répartissant en deux groupes (l'un d'ophtalmies du nouveau-né, l'autre d'UNG) on a obtenu 6 isolements de Chlamydia appartenant à un sérotype du sous-groupe A récemment défini : le TRIC Type G.

Dans tous les cas, l'agent fut isolé des parties génitales ou du rectum ou de l'oeil infecté en association avec une infection génitale, comme ceci survient avec l'agent TRIC de Type D, E, ou F. 